\title{
Final Results of a Phase II Study of Bevacizumab, Cisplatin and Pemetrexed as First-Line Therapy for Patients with Advanced Non-Squamous Non-Small Cell Lung Cancer
}

\author{
Guillermo López Vivanco, Eider Azkona, Sergio Carrera, Aintzane Sancho, \\ Itziar Rubio, Juan Manuel Mañé, Begoña Calvo, Unai Aresti, \\ Aitziber Buque, Inés Marrodán, Alberto Muñoz \\ Servicio de Oncología Médica, Hospital de Cruces, Bilbao, Spain \\ Email: guillermo.lopezvivanco@osakidetza.eus
}

Received 13 April 2016; accepted 24 June 2016; published 27 June 2016

Copyright (C) 2016 by authors and Scientific Research Publishing Inc.

This work is licensed under the Creative Commons Attribution International License (CC BY). http://creativecommons.org/licenses/by/4.0/

\section{(c) (i) Open Access}

\section{Abstract}

Background: Efficacy and safety data for cisplatin and pemetrexed plus bevacizumabinnon squamousnon non-small cell lung cancer (NSCLC) are still limited. Nevertheless, either bevacizumab plus platinum doublet or pemetrexed plus platinum is approved options for first line therapy. Predictive factors for bevacizumab are needed. KRAS is one of the most common oncogenic drivers in lung cancer. Its prognostic and predictive value in NSCLC is under investigation. Patients and methods: This trial evaluates the addition of bevacizumab $7.5 \mathrm{mg} / \mathrm{kg}$ to cisplatin $75 \mathrm{mg} / \mathrm{m}^{2} \mathrm{plus}$ pemetrexed $500 \mathrm{mg} / \mathrm{m}^{2}$ as first line treatment in stage IV non-squamous NSCLC patients. Maintenance bevacizumab was received as monotherapy until progressive disease, unacceptable toxicityor consent with drawal. The primary objective was progression free survival (PFS). Secondary objectives included overall survival (OS), safety, global objective responses and the determination of KRAS mutation at baseline. Results: From March 2009 to March 2012, 31 patients were enrolled. Mean age was 59.19 (standard deviation (SD) 8.53). From all the patients included in this trial, $67.70 \%$ were men. KRAS was wild type in 19 patients $(58.06 \%)$; in $7(22.58 \%)$ was mutated and was unknown in 6 patients $(19.35 \%)$. Median PFS for KRAS mutated patients was 4 months, whereas for the KRAS wild type it was 7.9 months $(\mathrm{P}=0.0031)$. Median OS was 4 months for the KRAS population, and 16.1 months for the KRAS wild type $(\mathrm{P}=\mathbf{0 . 0 0 3 2})$. Twenty four patients $(77.42 \%)$ experienced at least a grade 3 - 4 adverse event. The most common grade 3 - 4 toxicity was asthenia. Conclusions: Both PFS and OS were statistically longer for the KRAS wild type patients com- 
pared with the $K R A S$ mutated population $(\mathrm{P}=0.0031)$. Median OS was shorter than the reported in previous trials with bevacizumab. Nevertheless, focussing on the OS for KRAS wild type patients, this achieves a result or $\mathbf{1 6 . 1}$ months. Therefore, this would be a consistent data supporting to qualify this parameter as a predictive factor before starting treatment for NSCLC.

\section{Keywords}

\section{Non-Small-Cell Lung Cancer, Bevacizumab, Pemetrexed, Predictive Biomarker, KRAS}

\section{Introduction}

Advanced non-small cell lung cancer (NSCLC) has a poor prognosis, with a 5 -year survival rate $<15 \%$ in spite of diagnostic and therapeutic tools; existing therapies have limited activity and considerable toxicity [1] [2]. Estimated ratio morbidity/incidence is 0.9 [3]. Therapeutic approach is chosen depending on several factors such as functional status and comorbidity. So far today, systemic therapy is the only treatment that has shown that improves survival and quality of life [4].

The role of VEGF in stimulating tumor angiogenesis, maintaining existing vasculature and resistance to traditional therapies, with his negative prognostic significance in NSCLC, makes it an important therapeutic target in solid tumors [5]. Bevacizumab targets the vascular endothelial growth factor (VEGF) that has demonstrated improvement in clinical outcomes for multiple tumor types when combined with chemotherapy [5] [6]. In patients with advanced NSCLC bevacizumab treatment is associated to increase survival in the population when added to cytotoxic chemotherapy, which has led to its incorporation in neoadjuvant and adjuvant NSCLC clinical.

Pemetrexed has shown to have high response rates in NSCLC in Phase II and III trials, as monotherapy in the second line setting, combined with cisplatinum as first line treatment, and as a maintenance therapy. In combination with cisplatinum, pemetrexed has demonstrated an equivalent efficacy with a better safety profile when compared with cisplatinum-gemcitabine in patients with non-squamous NSCLC, with a more suitable posology [7]. Based on these results, this combination has been approved as first-line treatment of malignant pleural mesothelioma and non-squamous NSCLC patients [8].

When chemotherapy is indicated, platinum doublet chemotherapy may be considered for "fit" non-squamous NSCLC patients, either alone or combined with bevacizumab. Platinum chemotherapy plus bevacizumab was approved by the Food and Drug Administration (FDA) for non-squamous NSCLC treatment as first-line therapy [8]. This decision was based in Sandler's clinical trial studying carboplatinum/paclitaxel with or without bevacizumab. The median overall survival (OS) for the combination carboplatin/paclitaxel and bevacizumab was 12.3 months as compared with 10.3 months in the chemotherapy-alone group (hazard ratio for death, $0.79 ; \mathrm{P}=$ 0.003). Median progression-free survival (PFS) was also better in the bevacizumab arm (6.2 versus 4.5 months [9].

Following these results, a phase IIclinical trial was published analyzing survival and safety of bevacizu$\mathrm{mab} /$ carboplatin/paclitaxel treatment versus cisplatin/pemetrexed. PFS and OS were comparable between both arms, but the arm with bevacizumab showed a worse safety profile in terms of neuropathy $(P=0.06)$, deep vein thrombosis $(\mathrm{P}=0.23)$, proteinuria $(\mathrm{P}=0.23)$, and hypertension $(\mathrm{P}=0.11)[10]$.

Nevertheless, these two combinations obtained similar results in terms of survival, and may be considered as standard treatments for non-squamous NSCLC. Lack of predictive factors is still present despite treatment selection depending on them could probably be helpful to select those patients who are going to have benefit from a therapy.

The observed antitumor activities of bevacizumab with cisplatinum-pemetrexed, and their different mechanisms of action provide the rationale for evaluating the combination of these three agents in patients with advanced NSCLC in a single center, single-arm, open label, phase II study at the Cruces hospital in Spain.

On the other hand, RAS gene family (HRAS, NRAS and KRAS) is one of the most frequently oncogenes altered in human cancers [11] [12]. In NSCLC patients, the proteins encoded by these genes are assembled together, forming a protein structure with a GTP-ase activity, which participates in the signal transduction pathway of cell growth and differentiation [13]. Mutation in KRAS is seen in $15 \%$ to $25 \%$ of patients with NSCLC. Mutated p21 
proteins constitutively activate and stimulate growth and differentiation autonomously. These mutations have been frequently observed in various tumor types, primarily in colorectal, pancreatic and lung, The improvement in molecular technology has led to development of various techniques for molecular diagnostics and as well as some targeted therapies [14] [15]. Unless there has been limited success in inhibiting the protein directly, phase 2 and phase 3 clinical trials have demonstrated success in inhibiting downstream effectors, specifically $M E K 1$ and/or $M E K 2$ with selumetinib and trametinib (albeit with poor tolerability). The prognostic and predictive value of KRAS mutations in NSCLC still needs to be demonstrated.

\section{Patients and Methods}

\subsection{Patient Eligibility}

Patients with previously untreated advanced non-squamous NSCLC were enrolled. Additional inclusion criteria included Eastern Cooperative Oncology Group (ECOG) performance status $<2$, age $>18$ years, expected life expectancy $>3$ months, adequate renal, hepatic, coagulation and hematologic function. Informed consent form was provided to all patients before any procedure was performed. Patients were not eligible if they had received any prior treatment for advanced disease, had a history of abdominal fistula or gastrointestinal perforation or esophageal varices, significant vascular, and/or bleeding disorders, hypertension or if they had evidence of other serious illness or condition, including cardiac disease, congestive heart failure, psychiatric disorder or active infection, or open bone fracture, if they had a grade 2 or higher neurotoxicity, or a recent acetylsalicylic acid treatment or oral anticoagulants. Patients were also excluded if they had had another cancer, except basal-cell carcinoma of the skin or in situ cervical cancer within the previous 5 years or if the patient was a pregnant or breastfeeding woman. Other exclusion criteria included major surgery or open biopsy within the previous 4 weeks, serious non-healing wound, ulcer or fracture or other experimental or antitumor therapy within the previous 30 days, brain metastases or any other uncontrolled brain pathology, invasion of central vessels known by imaging tests.

\subsection{Treatment Plan and Dose Modifications}

All patients included in this trial received an intravenous infusion of bevacizumab $7.5 \mathrm{mg} / \mathrm{kg}$, pemetrexed 500 $\mathrm{mg} / \mathrm{m}^{2}$ and cisplatin $75 \mathrm{mg} / \mathrm{m}^{2}$ sequentially on Day 1 of each 21-day cycle. Pemetrexed was administered over 10 minutes. Cisplatin was administered within 1 to 3 hours assuring the correct hyperhydration of the patient. Treatment was administered for up to six cycles. Those achieving response or stable disease received maintenance bevacizumab $7.5 \mathrm{mg} / \mathrm{kg}$ once every 3 weeks until disease progression, unacceptable toxicity or patient decision. Pemetrexed-treated patients received standard supplementation with folic acid orally (350 to $1000 \mathrm{mcg}$ daily), vitamin B12 intramuscularly (1000 mcg once every three cycles), and dexamet has one prophylaxis orally (4 mg twice per day) as preventive medication throughout the study duration.

In case of grade $\leq 2$ adverse events (AEs), symptomatic treatment was given with no modification of doses. If grade $\geq 3$ AEs occurred, pemetrexed and cisplatin were withheld until resolution to grade 1 , and thereafter, doses were reduced.

In cases of hematologic toxicity, treatment with cisplatin and pemetrexed could be delayed for up to 2 weeks until the day 1 absolute neutrophil count (ANC) was $\geq 1.5 \times 10^{9} / \mathrm{L}$ and platelet count was $>100 \times 10^{9} / \mathrm{L}$. Pemetrexed doses could then be reduced to $75 \%$ or $50 \%$ of the previous dose, depending on the ANC and platelet nadirs. Cisplatin dose modifications were not allowed due to hematologic toxicity. In case of anaemia, patients were given the adequate support treatment. In cases of grade $\geq 3$ nonhematological toxicities (except for alopecia and neurotoxicity), pemetrexed and cisplatin were withheld until resolution and then reduced to $75 \%$ or $50 \%$ (for grade $3-4$ mucositis) of the previous doses. Cisplatin or pemetrexed treatment was withheld if grade $>1$ neurotoxicity; persistence of grade $>1$ neurotoxicity for more than 2 weeks required the study discontinuation for the patient. Cisplatin was discontinued if grade $>3$ auditory loss. For liver toxicity, only modification of pemetrexed dose was allowed.

\subsection{Efficacy Assessment}

$K R A S$ mutational status was determined at the beginning of the study. VEGF levels were assessed before 1 st cycle and every 2 cycles until treatment end and at progression. 
After baseline evaluation, patients were evaluated radiographically [Thoracic X-ray, computed tomography (CT) or CT plus Positron Emission Tomography (PET), PET (optional) or Magnetic Resonance Imaging (MRI)] every 2 cycles during induction and maintenancetreatment. Safety was evaluated at each cycle, and efficacy was assessed every other cycle.

\subsection{Statistical Considerations}

Based on Scagiotti's study [7], PFS at 1 year was less than 10\% for the cisplatin-pemetrexed group, corresponding to a PFS 4.8 months. To estimate the sample size, Fleming's single stage procedure for phase II studies was followed, which is based on a minimum efficacy percentage $\left(\pi_{(0)}\right)$. Under this minimum value treatment would be considered as treatment failure. A unilateral contrast was estimated $\alpha=0.05$ and $\beta$ error $=0.2$. With these assumptions, having as minimum value $\pi_{(0)}=0.08$ and as an optimal value $\pi_{(1)}=0.23,28$ evaluable patients were needed.

Survival analyses were done in the Intention-to-treat (ITT) and in the per-protocol (PP) population. Overall response rate (ORR) analysis was done in the ITT and in the evaluable population. ITT population comprised all patients included in the study. Population included the patients in the ITT population with no major protocol violations and who had received at least one dose of the study treatment. All patients with measurable disease who meteligibility criteria and received at least 2 cycles of combination treatment were evaluable for response (evaluable population). All enrolled patients who received the study drug were included in the safety analysis.

The primary endpoint was PFS. Based in the study of Scagliotti et al. (7), PFS at one year was $<10 \%$ for the cisplatin plus pemetrexed group, which corresponds to a median PFS of 4.8 months. According to Fleming's single stage procedure [10] a minimum foreseen 28 evaluable patients were required considering that the minimal percentage of efficacy is 0.08 and the optimal efficacy value is 0.23 . This design provided an alpha error of $\alpha=$ 0.05 (one-sided) and a beta error of $\beta=0.2$ (80\% power).

Secondary endpoints included OS, ORR, and toxicity. In addition, KRAS mutations before treatment start and serum level of VEGF at baseline, every two cycles during treatment period and at progression were analyzed as secondary variables.

PFS was considered as the time interval from date of inclusion until the date of disease progression or death due to any cause (whichever occurs first). OS was measured from date of inclusion until death. PFS and OS were summarized by Kaplan-Meier curves.

\section{Results}

\subsection{Patient Characteristics}

From March 2009 to March 2012, 31 patients (out of the 37 patients screened) were enrolled into the study and constituted the ITT population. The median patient age was 59 years. Focusing on gender $67.7 \%$ were men and $32.3 \%$ were women. Most of the patients presented ECOG 1(83.9\%). Thirty patients had adenocarcinoma and one patient had a large cell carcinoma. Disease was stage IV in all cases. The most common metastases location was lung (23.53\%), followed by lymph nodes $(22.35 \%)$ and bone $(20 \%)$.

Patients' characteristics are summarised in Table 1.

\subsection{Treatment Exposure}

All ITT population (31 patients) received at least one cycle of treatment with a median of 4 induction cycles per patient $(\mathrm{Q} 1=2 ; \mathrm{Q} 3=6)$. Fifteen patients $(48.4 \%$ of the safety population) received at least 6 cycles of maintenance therapy, and 3 patients $(9.7 \%)$ received 12 or more cycles. Seventeen patients $(54.84 \%)$ stopped receiving the study drug due to progression disease; 10 patients $(32.36 \%)$ stopped receiving the study drug due to adverse event (4 patients died prematurely due to comorbidity).

\subsection{Efficacy}

Of the total 31 patients enrolled in this trial, only 24 were evaluable for efficacy (as they received $\geq 2$ cycles of treatment) and did not present major protocol violations. KRAS was wild type in $58.06 \%$ patients, mutated in $22.58 \%$ and unknown in $19.35 \%$.

Nearly half of the patients in the ITT population (48.4\%) had a partial response (none complete response was 
Table 1. Patient Demographics $(\mathrm{N}=31)$.

\begin{tabular}{|c|c|c|c|}
\hline \multirow{2}{*}{ Characteristics } & & \multicolumn{2}{|c|}{ Patients } \\
\hline & & No. & $\%$ \\
\hline \multicolumn{4}{|l|}{ Gender } \\
\hline Men & & 21 & 67.7 \\
\hline \multicolumn{4}{|l|}{ Age (years) } \\
\hline Median (range) & $59.0(42.0-74.0)$ & & \\
\hline \multicolumn{4}{|l|}{ Performance Status (ECOG) } \\
\hline 0 & & 5 & 16.1 \\
\hline 1 & & 26 & 83.9 \\
\hline \multicolumn{4}{|l|}{ Disease stage } \\
\hline stage IV & & 31 & 100.0 \\
\hline \multicolumn{4}{|l|}{ metastases } \\
\hline lung & & 20 & 64.5 \\
\hline nodes & & 19 & 61.2 \\
\hline Bone & & 17 & 51.8 \\
\hline Liver & & 13 & 41.9 \\
\hline Other (kidney, adrenal gland, etc.) & & 16 & 51.6 \\
\hline \multicolumn{4}{|l|}{ Histology } \\
\hline Adenocarcinoma & & 30 & 96.8 \\
\hline Large-cell carcinoma & & 1 & 3.2 \\
\hline \multicolumn{4}{|l|}{ KRAS mutation } \\
\hline mutated & & 7 & 22.6 \\
\hline wild-type & & 18 & 58.1 \\
\hline unknown & & 6 & 19.4 \\
\hline
\end{tabular}

ECOG: Eastern Cooperative group.

achieved), and $25.8 \%$ had stable disease; therefore, clinical benefit rate was $74.2 \%$. Four patients $(12.90 \%)$ developed progressive disease (PD) at first tumor assessment during treatment and 4 patients were non-evaluable. Median PFS was 7.2 months for the ITT population (CI 2.9 - 8.1); median PFS for mutated KRAS patients was 4 months, whereas it was 7.9 months for $K R A S$ wild type patients, reaching this difference the statistical significance $(\mathrm{P}=0.0031)$. The Kaplan Meier plots of PFS are shown in Figure 1.

\subsection{Overall Survival (OS)}

OS was a prespecified secondary endpoint. An analysis of the OS was performed based on 25 death events ( $80.65 \%$ of the ITT population) at the time of the data cut-off for the final PFS analysis.

Median OS was 11.3 months for the ITT population (95\% CI, 4.6 - 17.8), 4 months for the KRAS mutated patients, and 16.1 months for those with $K R A S$ wild type $(\mathrm{P}=0.0032)$, with a Hazard Ration (HR) 4.318, showing that there is a higher death associated to the presence of mutation in KRAS gene.

In the global ITT population, $16.13 \%$ of all patients were alive at 1 year, and $6.45 \%$ at 18 months Focusing on KRAS mutational status, OS at 1 year was $22.22 \%$ for $K R A S$ wild type versus $0 \%$ for $K R A S$ mutated patients.

The Kaplan Meier plots of OS are shown in Figure 2.

\subsection{Toxicity}

All 31 patients were included in the safety analysis as they received at least one cycle of treatment.Among 31 patients assessed for toxicity, all of them experienced at least $1 \mathrm{AE}$ (most were Grade 1 or 2). Twenty four patients $(77.42 \%)$ experienced at least a grade 3 - 4 AE. The most common grade $3-4$ toxicity was asthenia. Three patients had a pulmonary embolism and three had a deep vein thrombosis. Grade 3 or 4 neutropenia occurred in $4(12.9 \%)$ patients. Adverse events of interest with bevacizumab were primarily grade 1 or 2 . Three patients (9.7\%) had grade 3 hypertension and one patient (3.2\%) had cardiac infarction.

\section{Discussions}

At present, treatment options for non-squamous NSCLC EGFR wild type patients (without any actionable or 


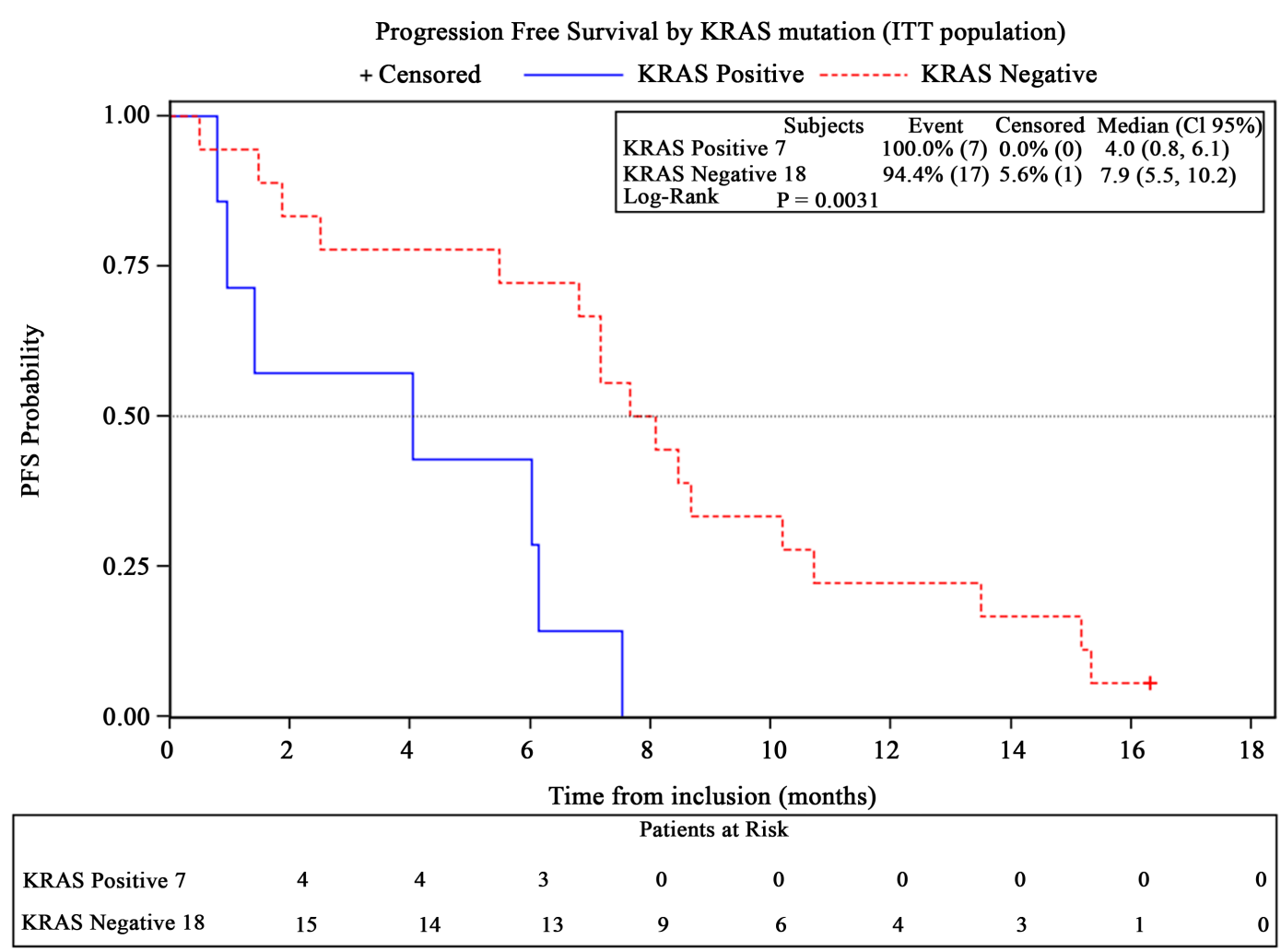

Figure 1. PFS according to $K R A S$ status.

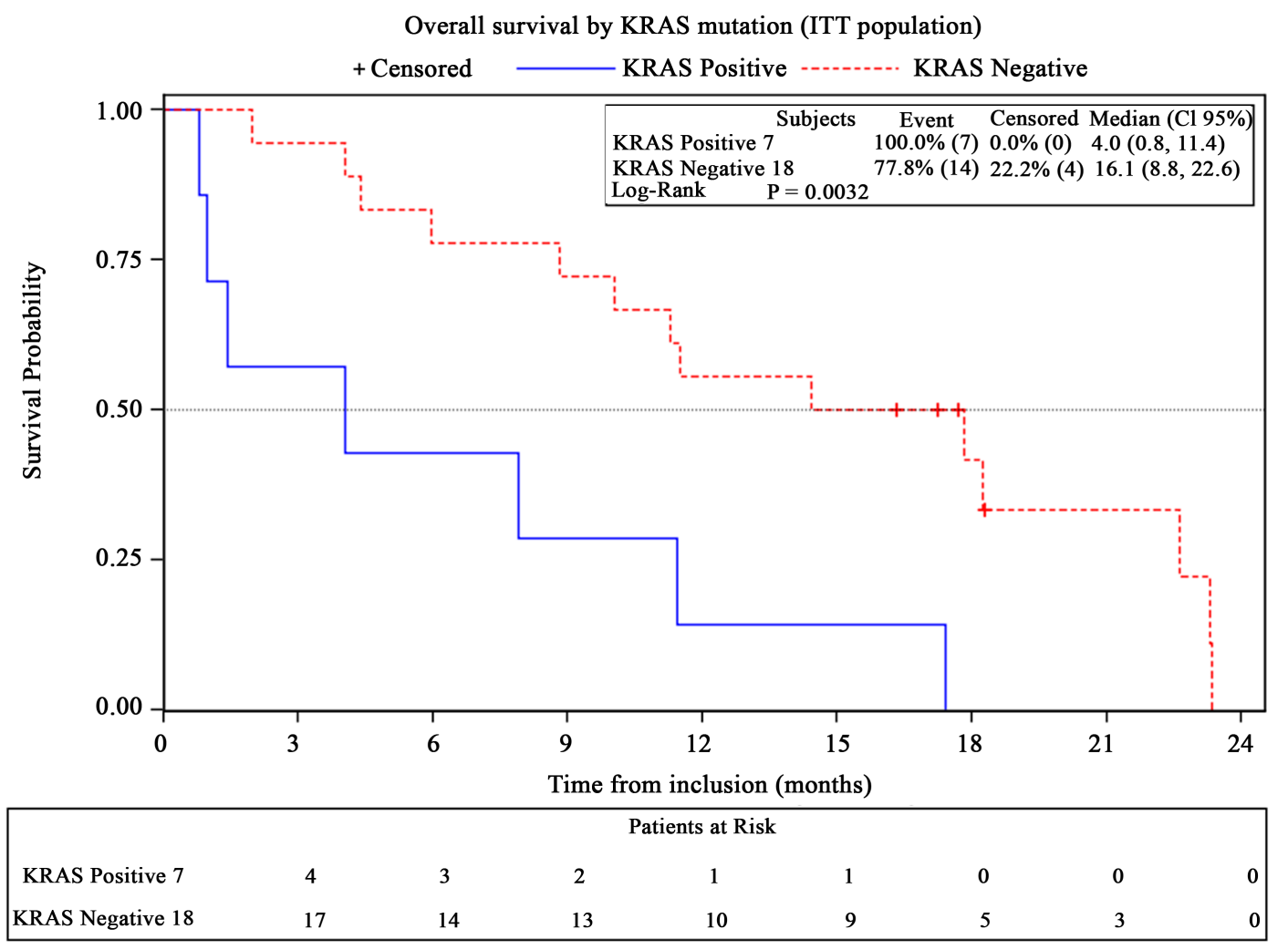

Figure 2. OS according to $K R A S$ status. 
driver mutation) include a platinum doublet with or without bevacizumab. The goals in the management are improving quality of life and overall survival. Median OS with current treatment option is between 11 - 15 months [8].

Chemotherapy based on platinum doublets plus bevacizumab may be a good choice for fit patients [16]. This is supported by a meta-analysis published in 2011 in which five phase II and phase III randomized clinical trials evaluating the efficacy of the addition of bevacizmab to different chemotherapy regimens were included, most of them in first line treatment [12]. The administration of bevacizumab was associated to a significant increase in median OS (HR 0.89; 95\% CI 0.79 to 0.99; $\mathrm{P}=0.04$ ), PFS (HR 0.73; 95\% CI 0.66 to $0.82 ; \mathrm{P}<0.00001$ ) and response rate (OR 2.34; $95 \%$ CI 1.89 to $2.89 ; \mathrm{P}<0.00001$ ) with an $11 \%$ reduction in the risk of death. However, hypertension, febrile neutropenia and bleeding were more frequent in patients receiving bevacizumab, with a small, but statistically significant, increase in deaths with bevacizumab (OR 1.82, 95\% CI 1.04 to 3.18 ; all trials).

These positive results of the addition of bevacizumab to a platinum doublet were corfirmed by the study or Crinò and colleagues [17]. This is a phase IV trial in which 2.012 stage III-B and IV untreated non-squamous NSCLC patients were treated with bevacizumab plus standard chemotherapy up to 6 cycles and, in case of no progressive disease, a maintenance period with bevacizumab monotherapy. The incidence of clinically significant (grade $>$ or $=3$ ) adverse events of special interest was generally low, being the most frequent thromboembolism in $172(8 \%)$ patients, hypertension in $125(6 \%)$, bleeding in $80(4 \%)$, proteinuria in $67(3 \%)$, and pulmonary haemorrhage in $15(1 \%) .57(3 \%)$ patients died because of these adverse events Authors conclude that the combination of bevacizumab with different chemotherapy schemes used as clinical standard practice was safe and well tolerated.

There are also results from a phase III trial designed to compare bevacizumab with or without pemetrexed as maintenance treatment after induction with the triplet combination. Showing statistically significant differences inedian PFS favoring the experimental arm (3.7 and 7.4 months for the bevacizumab alone and bevacizumab plus pemetrexed arms, respectively (HR 0.48; 95\% CI, 0.35 to 0.66 ; P < 0.001) [18]. PFS after first induction treatment was improved from randomization, with medians of 6.6 months (95\% CI, 6.0 to 7.8 months) in the bevacizumab arm and 10.2 months $(95 \% \mathrm{CI}, 9.1$ to 11.7 months) in the bevacizumab plus pemetrexed arm. There was a more evident benefit in those patients achieving a PR after induction (3.9 v 8.6 months; HR, 0.42; $95 \% \mathrm{CI}, 0.28$ to $0.64 ; \mathrm{P}<0.001)$ and also in the patients that had achieved SD (3.3 v 6.8 months; HR, $0.63 ; 95 \%$ CI, 0.41 to $0.97 ; \mathrm{P}=0.036)$.

Our median OS has been shorter than that reported in other clinical trials with bevacizumab (included the study testing the combination with pemetrexed) but, focussing on the OS for $K R A S$ wild type patients, this achieves a result or 16.1 months. Therefore, this would be a consistent data supporting to qualify this parameter as a predictive and prognostic factor before starting treatment for NSCLC. This benefit is also present for the PFS result, with a statistically significant difference between mutated and wild type populations.

The safety profile when bevacizumab is added to chemotherapy is to be considered. It increases the treatment-related mortality, as found in meta-analyses in different sort of solid tumors [19] [20]. The adverse events that were reported in our trial were consistent with those related to all three agents, administered in conjunction, but the rate of serious adverse events was higher than expected: pulmonary embolism in $9.7 \%$ of patients, deep vein thrombosis in $9.7 \%$ of patients, cardiac infarction in $3.2 \%$ of patients and grade III or IV neutropenia in $12.9 \%$. This should be specially taken into account in a disease as this with a very poor prognosis. In addition, $77.42 \%$ of patients experienced at least grade 3 - 4 adverse events (AEs).

\section{Conclusions}

In this phase II clinical trial conducted in untreated stage IV non-squamous NSCLC patients, the addition of bevacizumab to a combination treatment of cisplatin and pemetrexed resulted in PFS of 7.2 months and OS of 11.3 months, being PFS better than the reported in previous trials in this target population.

A statistically significant increase in both PFS and OS is seen in KRAS wild type patients as compared with KRAS mutated patients, indicating that $K R A S$ could become a predictive and prognostic marker. However, the analyzed sample was too small to draft definitive conclusions.

\section{Authors' Contributions}

All authors contributed equally and extensively to the work presented in this paper. G. López Vivanco designed 
the protocol. All authors included patients in the study, discussed the results and implications and commented on the manuscript at all stages.

\section{Acknowledgements}

Sonia Maciá (Pivotal SL) provided advice to properly handle and submit the paper.

\section{Funding}

Roche Pharma has supported funding for monitoring, data management, biostatistics and medical writing.

\section{Informed Consent}

All patients included in this study were clearly informed about the trial, and signed an informed consent form, that was previously reviewed and approved by local Ethics Committee.

\section{Research Involving Human Participants and/or Animals}

This research has been involved human participants and, as such, as been performed according to the terms stipulated and the ethical principles laid out in the latest version of the Declaration of Helsinki, the International Conference on Harmonisation (ICH) Good Clinical Practice (GCP) guidelines and applicable legal requirements.

\section{Conflict of Interest}

The authors have no relevant affiliations or financial interest in or financial conflict with the subject matter or materials discussed in the manuscript. This includes employment, consultancies, honoraria, stock ownership or options, expert testimony, grants or patents received or pending, or royalties.

\section{References}

[1] Berrino, F. (2003) The EUROCARE Study: Strengths, Limitations and Perspectives of Population-Based, Comparative Survival Studies. Annals of Oncology, 14, v9-v13. http://dx.doi.org/10.1093/annonc/mdg750

[2] Brabender, J., Park, J., Metzger, R., Schneider, P., Lord, R., Hölscher, A., et al. (2002) Prognostic Significance of Cyclooxygenase 2 mRNA Expression in Non-Small Cell Lung Cancer. Annals of Surgery, 235, 440-443. http://dx.doi.org/10.1097/00000658-200203000-00017

[3] Parkin, D.M., Bray, F., Ferlay, J. and Pisani, P. (2001) Estimating the World Cancer Burden: Globocan 2000. International Journal of Cancer, 94, 153-156. http://dx.doi.org/10.1002/ijc.1440

[4] American Society of Clinical Oncology (1997) Clinical Practice Guidelines for the Treatment of Unresectable Non-Small-Cell Lung Cancer. Journal of Clinical Oncology, 15, 2996-3018.

[5] Folkman, J., Merler, E., Abernathy, C. and Williams, G. (1971) Isolation of a Tumor Factor Responsible of Angiogenesis. The Journal of Experimental Medicine, 133, 276-288. http://dx.doi.org/10.1084/jem.133.2.275

[6] Ferrara, N. and Davis-Smyth, T. (1997) The Biology of Vascular Endothelial Growth Factor. Endocrine Reviews, 18, 4-25. http://dx.doi.org/10.1210/edrv.18.1.0287

[7] Scagliotti, G.V., Parikh, P., Von Pawel, J., Biesma, B., Vansteenkiste, J., Manegold, C., et al. (2008) Phase III Study Comparing Cisplatin Plus Gemcitabine with Cisplatin Plus Pemetrexed in Chemotherapy-Naive Patients with Advanced-Stage Non-Small-Cell Lung Cancer. Journal of Clinical Oncology, 26, 3543-3551. http://dx.doi.org/10.1200/JCO.2007.15.0375

[8] Sandler, A., Gray, R., Perry, M.C., Brahmer, J., Schiller, J.H., Dowlati, A., et al. (2006) Paclitaxel-Carboplatin Alone or with Bevacizumab for Non-Small-Cell Lung Cancer. The New England Journal of Medicine, 355, 2542-2550. http://dx.doi.org/10.1056/NEJMoa061884

[9] Kader, Y., Le Chevalier, T., El-Nahas, T. and Sakr, A. (2013) Comparative Study Analyzing Survival and Safety of Bevacizumab/Carboplatin/Paclitaxel and Cisplatin/Pemetrexed in Chemotherapy-Naïve Patients with Advanced Non-Squamous Bronchogenic Carcinoma Not Harboring EGFR Mutation. Onco Targets and Therapy, 6, 803-809.

[10] Fleming, T.R. (1982) One-Sample Multiple Testing Procedure for Phase II Clinical Trials. Biometrics, 38, $143-151$. http://dx.doi.org/10.2307/2530297 
[11] Aunoble, B., Sanches, R., Didier, E., et al. (2000) Major Oncogenes and Tumor Suppressor Genes Involved in Epithelial Ovarian Cancer (Review). International Journal of Oncology, 16, 567-576. http://dx.doi.org/10.3892/ijo.16.3.567

[12] Hruban, R.H., Iacobuzio-Donahue, C., Wilentz, R.E., et al. (2001) Molecular Pathology of Pancreatic Cancer. The Cancer Journal, 7, 251-258.

[13] Ellis, C.A. and Clark, G. (2000) The Importance of Being K-Ras. Cell Signaling, 12, 425-434. http://dx.doi.org/10.1016/S0898-6568(00)00084-X

[14] Keohavong, P., Gao, W.M., Zheng, K.C., et al. (2004) Detection of K-ras and p53 Mutations in Sputum Samples of Lung Cancer Patients Using Laser Capture Microdissection Microscope and Mutation Analysis. Analytical Biochemistry, 324, 92-99. http://dx.doi.org/10.1016/j.ab.2003.09.030

[15] Minamoto, T., Mai, M. and Ronai, Z. (2000) K-ras Mutation: Early Detection in Molecular Diagnosis and Risk Assessment of Colorectal, Pancreas, and Lung Cancers: A Review. Cancer Detection and Prevention, 24, 1-12.

[16] Peters, S., Adjei, A., Gridelli, C., Reck, M., Kerr, K. and Felip, F. (2012) Metastatic Non-Small-Cell Lung Cancer: ESMO Clinical Practice Guidelines. Annals of Oncology, 23, vi56-vi64.

[17] Lima, A.B., Macedo, L.T. and Sasse, A.D. (2011) Addition of Bevacizumab to Chemotherapy in Advanced Non-Small Cell Lung Cancer: A Systematic Review and Meta-Analysis. PLoS One, 6, e22681. http://dx.doi.org/10.1371/journal.pone.0022681

[18] Crino, L., Dansin, E., Garrido, P., Griesinger, F., Laskin, J., Pavlavis, N., et al. (2010) Safety and Efficacy of First-Line Bevacizumab-Based Therapy in Advanced Non-Squamous Non-Small-Cell Lung Cancer (SAiL, MO19390): A Phase 4 Study. The Lancet Oncology, 11, 733-740. http://dx.doi.org/10.1016/S1470-2045(10)70151-0

[19] Barlesi, F., Scherpereel, A., Rittmeyer, A., Pazzola, A., Ferrer Tur, N., Kim, J.H., et al. (2013) Randomized Phase III Trial of Maintenance Bevacizumab with or without Pemetrexed after First-Line Induction with Bevacizumab, Cisplatin, and Pemetrexed in Advanced Nonsquamous Non-Small-Cell Lung Cancer: AVAPERL (MO22089). Journal of Clinical Oncology, 31, 3004-3011. http://dx.doi.org/10.1200/JCO.2012.42.3749

[20] Ranpura, V., Hapani, S. and Wu, S. (2011) Treatment-Related Mortality with Bevacizumab in Cancer Patients: A Meta-Analysis. JAMA, 305, 487-494. http://dx.doi.org/10.1001/jama.2011.51

\section{Submit or recommend next manuscript to SCIRP and we will provide best service for you:}

Accepting pre-submission inquiries through Email, Facebook, Linkedin, Twitter, etc A wide selection of journals (inclusive of 9 subjects, more than 200 journals)

Providing a 24-hour high-quality service

User-friendly online submission system

Fair and swift peer-review system

Efficient typesetting and proofreading procedure

Display of the result of downloads and visits, as well as the number of cited articles

Maximum dissemination of your research work

Submit your manuscript at: http://papersubmission.scirp.org/ 